\title{
Stabilized Wide-Band Wavelength Conversion Enabled by CW-Triggered Supercontinuum
}

\author{
Xing Xu, Student Member, IEEE, Chi Zhang, T. I. Yuk, Kevin K. Tsia, Member, IEEE, \\ and Kenneth K. Y. Wong, Senior Member, IEEE
}

\begin{abstract}
We experimentally demonstrate a wide-band wavelength converter enabled by continuous-wave $(\mathrm{CW})$ triggered supercontinuum (SC) generated from a picosecond pulse source. In the presence of the trigger, the pulse-to-pulse SC stability considerably improved, leading to error-free wavelength conversion operations spanning from 1510 to $1615 \mathrm{~nm}$, which covers the $S$-, $\mathrm{C}$-, and L-bands of the ITU grids. In addition, the CW-triggered SC spectrum is significantly enhanced to over $300 \mathrm{~nm}$ measured at $20 \mathrm{~dB}$ below the peak spectral power (from $\sim 1400$ to $1700 \mathrm{~nm}$ and beyond). Our scheme thus provides a low pump power and wide-band solution for fiber-based optical wavelength conversion. With its better CW source, e.g., lower relative intensity noise and longer coherence length, the present scheme has the potential to be further applied to phase-modulated signals.
\end{abstract}

Index Terms-Optical signal processing, supercontinuum (SC) generation, wavelength conversion.

\section{INTRODUCTION}

$\mathbf{S}$ UPERCONTINUUM (SC), with its ultrabroadband spectrum generated from narrow-band pulses, is the versatile light source for a wide range of applications in both communication and bio-imaging, among which include ultrashort pulse generation, wavelength conversion, optical frequency metrology, and optical coherence tomography (OCT) [1], [2] . One effective scheme for ultrabroadband SC generation is based on primarily soliton fission, which happens when launching an intense pulsed pump into the nonlinear optical fiber in its anomalous dispersion regime [3]. However, as being initiated by modulation instability (MI), another competing process with soliton fission, can amplify the input noise, which in turns serves as the precursor of soliton fission. As a result, the soliton fission, and thus SC are initiated in a random fashion. To its extreme case, the rogue soliton (a rare but very intense event) [4] or the rogue void (a rare but very weak event) can be formed [5]. It makes the SC inherently incoherent and exhibit large pulse-to-pulse intensity

Manuscript received July 10, 2012; revised August 25, 2012; accepted September 5, 2012. Date of publication September 10, 2012; date of current version September 26, 2012. This work was supported in part by grants from the Research Grants Council of the Hong Kong Special Administrative Region, China, under Project HKU 7183/09E, Project HKU 7175/10E, and Project HKU 717911E, and the University Development Fund of The University of Hong Kong.

The authors are with the Department of Electrical and Electronic Engineering, University of Hong Kong, Hong Kong (e-mail: xuxing@ eee.hku.hk; zhangchi@eee.hku.hk; tiyuk@eee.hku.hk; tsia@eee.hku.hk; kywong@eee.hku.hk).

Color versions of one or more of the figures in this letter are available online at http://ieeexplore.ieee.org.

Digital Object Identifier 10.1109/LPT.2012.2218099

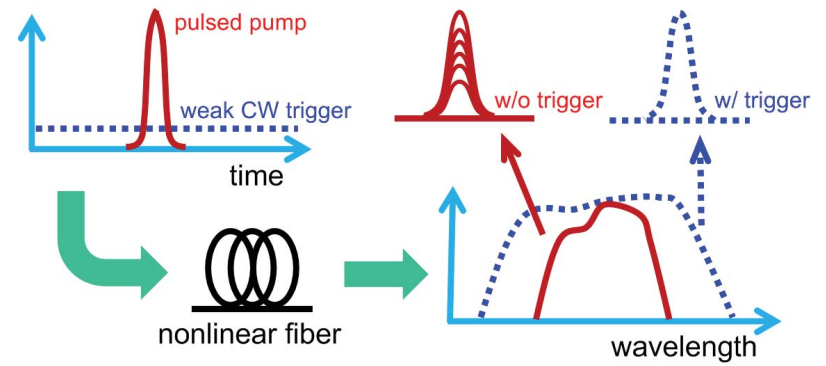

Fig. 1. Principle of the CW-triggered SC.

fluctuation - the pitfalls hampering the utility of SC in a wide range of applications [6]. This problem is particularly severe for wider input pulsewidth: picosecond or nanosecond [2]. Fortunately, it can be remedied by introducing a weak continuous-wave (CW) trigger together with the input pump pulse [7]. In this case, the MI is initiated by the controllable $\mathrm{CW}$ trigger, overwhelming the noise. Hence, the subsequent soliton fission would become more deterministic, making the SC more stable and coherent [8], [9]. Comparing to some former schemes which need either precise time delay control [9] or complex feedback process [10], this method provides a fairly simple and more effective solution to enhance both the covering range and the pulse stability of the generated SC. On the other hand, the presence of the $\mathrm{CW}$-trigger, locating within the MI gain bandwidth, could also accelerate the soliton fission process [8], [9], [11]. To its extreme case, the rogue soliton can be formed and the SC bandwidth can thus be drastically enhanced [7], [9].

As a result, the $\mathrm{CW}$-triggering scheme can be harnessed for realizing stable (low-noise) and wide-band wavelength conversion. This is in contrast to some former experiments [12]-[14], in which, only limited range of wavelength conversion (tens of nanometer) could be achieved. In our previous work [15], we have shown some preliminary results which confirmed the feasibility and advantages of our method. In the following parts of this letter, we first analyze the pulse-to-pulse stability improvement with the $\mathrm{CW}$ triggering, followed by a detailed demonstration of a wavelength converter based on the CWtriggered SC scheme. We demonstrate, to the best of our knowledge, for the first time that the stable SC can be restored from the rogue void condition by adding the $\mathrm{CW}$-trigger. The input pulsed pump was from a 10-Gb/s picosecond source with the pulsewidth of $6.3 \mathrm{ps}$. During the experiment, the bandwidth of the SC spectrum was increased to over $300 \mathrm{~nm}$ measured 


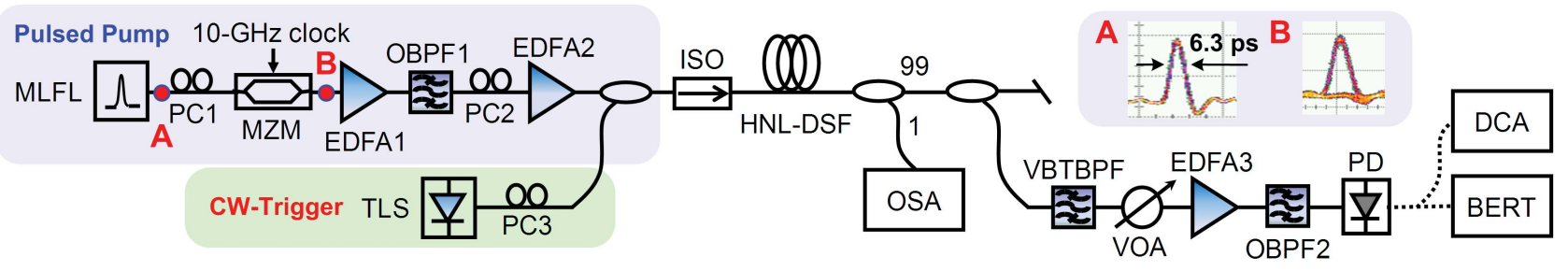

Fig. 2. Experimental setup. VOA: variable optical attenuator. PD: photodiode. (Other abbreviations are defined in the text.)

at $20 \mathrm{~dB}$ below the peak spectral power (from $\sim 1400$ to $1700 \mathrm{~nm}$ and beyond), while error-free (less than 3.8-dB power penalty at the bit-error rate (BER) of $10^{-9}$ ) operations were attained for over 100-nm spectrum range spanning S-, C- and L-band.

\section{PRinciple AND ExPERIMENTAL SetuP}

The principle of the CW-triggered picosecond SC is illustrated in Fig. 1. The process is realized by bringing in an external weak $\mathrm{CW}$ trigger which is co-propagating with the picosecond pulsed pump inside the fiber. Hence, the generated SC spectrum can be significantly broadened if the wavelength of the trigger locates at the MI gain peak [7]-[9]. As explained previously in the introduction, the MI growth here is initiated by the trigger rather than the noise, which leads to the stabilized soliton fission process during the SC generation. As a result, in addition to the spectrum broadening, the pulse-topulse instability is also improved, which can be visualized by the clear open eye-diagram comparing to the non-trigger case (right portion of Fig. 1).

The experimental setup is shown in Fig. 2. The pulsed pump, located at $1554.77 \mathrm{~nm}$, was generated from a modelocked fiber laser (MLFL) and intensity-modulated by a Mach-Zehnder modulator (MZM) driven by $10-\mathrm{GHz}$ clock signal. While the polarization controller (PC1) was utilized to align the polarization of the pulse with the transmission axis of the MZM. Two erbium-doped fiber amplifiers (EDFA) were applied to amplify the average pump power upto $365 \mathrm{~mW}(\sim 10.43 \mathrm{~W}$ peak power) before the highly-nonlinear dispersion-shifted fiber (HNL-DSF), which has the length $L=$ $150 \mathrm{~m}$, zero dispersion wavelength (ZDW) $\lambda_{0}=1554.7 \mathrm{~nm}$ and nonlinear coefficient $\gamma=30 W^{-1} \mathrm{~km}^{-1}$. The optical bandpass filter (OBPF), with 3-nm bandwidth between the two EDFAs, was used to minimize the amplified spontaneous emission (ASE) noise from the EDFA1. On the trigger branch, one CW tunable laser source (TLS), with the wavelength at $1613 \mathrm{~nm}$, was utilized. The power level of the trigger was more than 40-dB lower than the pump. After combining with a WDM coupler, the pulsed pump and the weak trigger were launched together into the HNL-DSF. At the fiber output, after removing the residual pump, the wavelength converted pulsed signal was filtered out by a variable bandwidth tunable bandpass filter (VBTBPF).

\section{EXPERIMENTAL Results AND Discussion}

The power stability improvement was quantified by the pulse-to-pulse amplitude histograms (Fig. 3). The filtered SC

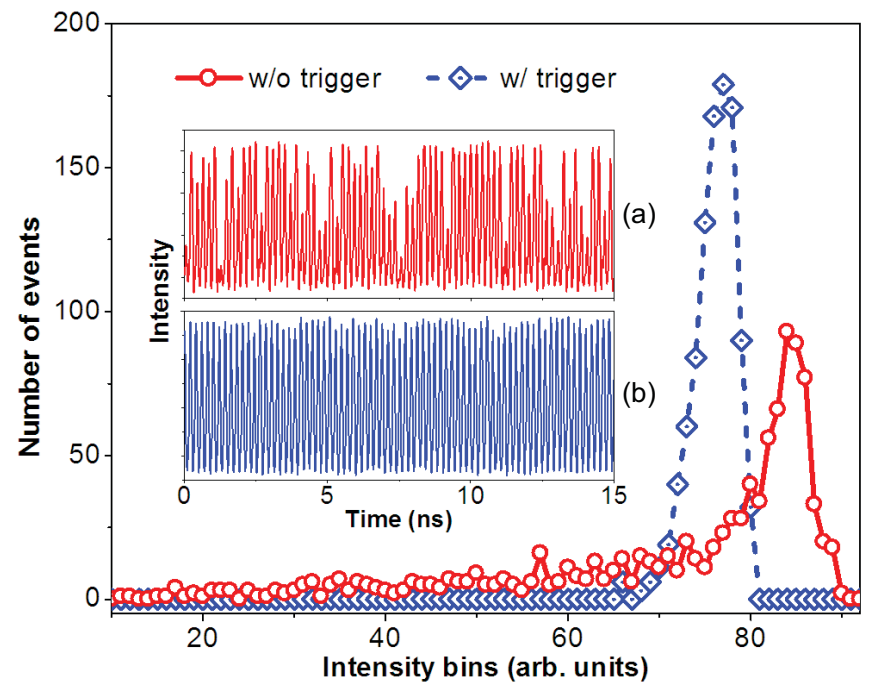

Fig. 3. Amplitude histograms for sliced SC @ 1566.82 nm without (red circle) and with (blue diamond) trigger. Insets: real-time pulse traces (a) without and (b) with trigger.

pulse traces (1000 pulses) were obtained from a real-time oscilloscope ( $4 \mathrm{GHz}, 20 \mathrm{GS} / \mathrm{s})$ as shown in the insets of Fig. 3 . From the figure of the non-trigger case (Fig. 3, red circle), it shows a clear left-skewed long-tail distribution, which resembles the occurrence of the optical rogue voids [4]. Indeed, there exists some rare and weak pulses in the pulse train as shown in the inset of Fig. 3. We note that this rare suppression is in contrast to our previous demonstration in which right-skewed extreme value statistic was observed [7]. Such difference is partly attributed to that the histograms in the two cases were obtained in different wavelength ranges of the SC. It has been shown that the temporal statistic of the SC shows dramatic variation across the whole SC spectrum [16], [17]. Another factor affecting the SC's power statistics is the pump power. Specifically, when the pump power exceeds a threshold beyond which soliton fission occurs during the SC generation, the power of the SC pulses (the longer-wavelength side of the SC) exhibit a left-skewed statistic (associated with rogue voids). In contrast, below this threshold, a right-skewed statistic (a signature of the existence of rogue waves) can be observed [5]. In our experiment, the pump power was measured to be higher than the threshold power, which was $\sim 280 \mathrm{~mW}$ ( $\sim 8$-W peak power). Therefore, the generated SC here shows the left-skewed statistic. Based on this result as well as our previous works [7], [8], we observe that stimulating the SC with a CW-trigger suppresses rogue waves (rogue voids) when the pump power is lower (higher) than the threshold. This 

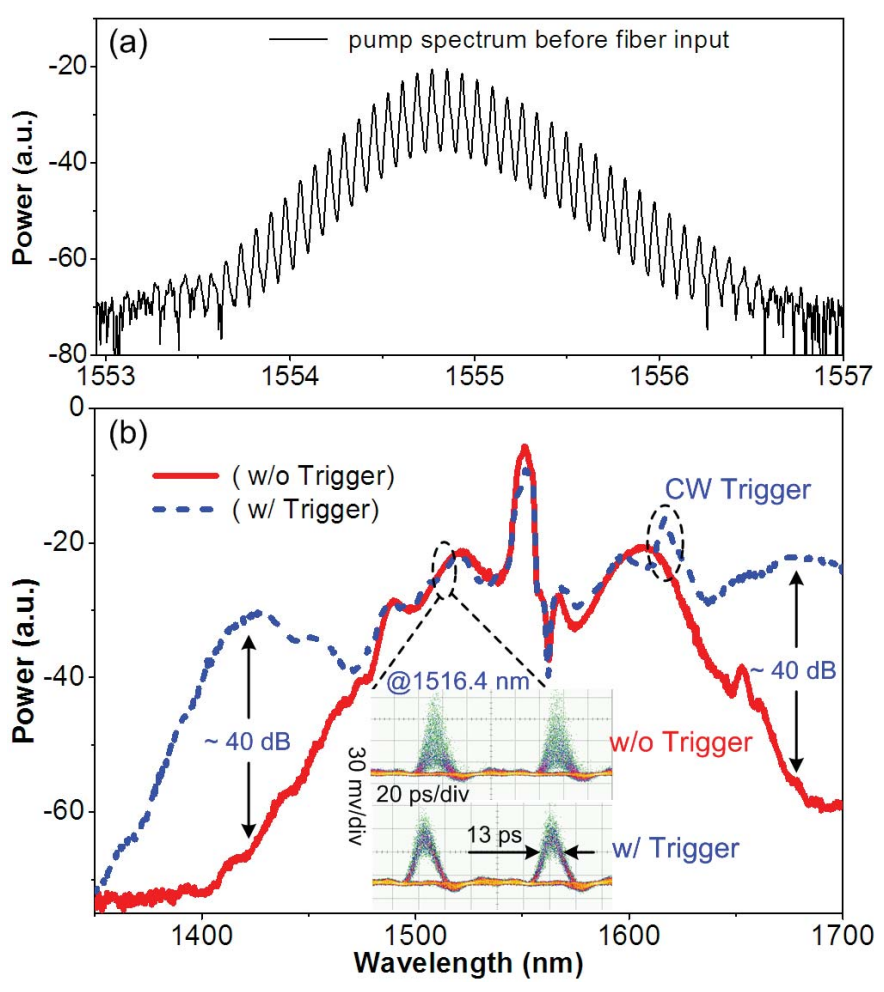

Fig. 4. (a) Pump spectrum before the fiber input (OSA resolution bandwidth: $0.02 \mathrm{~nm}$ ). (b) SC spectra: without (red solid line) and with (blue dotted line) trigger. Insets: eye-diagrams @ 1516.4 nm (OSA resolution bandwidth: $0.06 \mathrm{~nm})$.

is because both rogue waves and rogue voids are caused by spontaneous MI (i.e. noise), which is now overwhelmed by the controlled CW-trigger. Moreover, the temporal stability could also depend on a multitude of parameters, such as pump wavelength, power, pulse width and so on. Further extensive investigation, which takes all these parameters into account and studies how the interplay among them results in different temporal statistics, should be carried out in order to establish a more complete understanding of the entire SC dynamics.

When the CW trigger was introduced, the amplitude statistics was changed into an clear quasi-Gaussian distribution (Fig. 3, blue diamond) with $\sim 75 \%$ reduction in the standard deviation comparing to non-trigger case. Such improvement in pulse-to-pulse stability is also readily visualized from the realtime pulse traces (Fig. 3, insets). The small pulse amplitude variation in the triggered case can be attributed mainly to the relative intensity noise (RIN) and the limited coherence length $(\sim 30 \mathrm{~m})$ of our $\mathrm{CW}$ laser source. The former one could certainly contaminate the SC temporal stability, and the latter one degrades the phase coherence of the pulses [7]. Thus, a CW trigger with low RIN and longer coherence length (comparable to fiber length, in our experiment $\sim 150 \mathrm{~m}$ ) are favorable, especially for the potential applications of wavelength conversion or multicasting of phase-modulated pulse signals.

Results shown above to some extent illustrates the pulse stability enhancement with the $\mathrm{CW}$ trigger. In order to get a complete picture, we further investigated the wavelength conversion performance based on the $\mathrm{CW}$-triggered SC. The
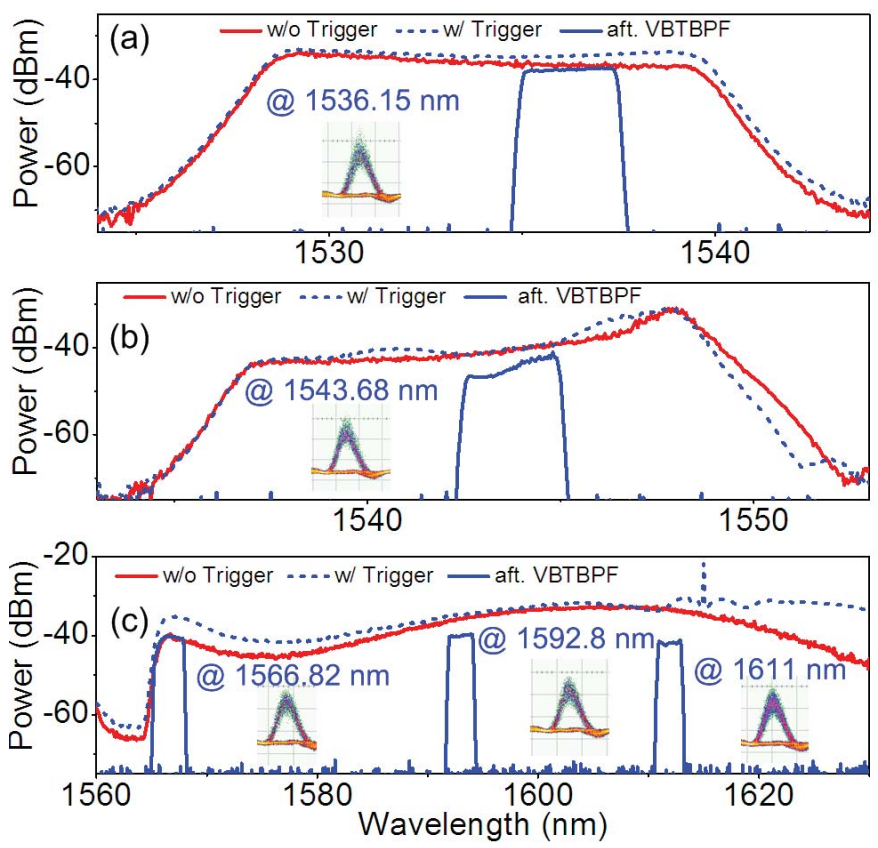

Fig. 5. SC spectra after WDM couplers without (red solid line) and with (blue dotted line) trigger, and after VBTBPF (blue solid line). (a) and (b) C-band: 1536.15 and $1543.68 \mathrm{~nm}$. (c) L-band: 1566.82, 1592.8 and $1611 \mathrm{~nm}$ (OSA resolution bandwidth: $0.06 \mathrm{~nm}$ ).

10-GHz clock train was substituted with $2^{31}-1$ pseudorandom binary sequence (PRBS) in Fig. 2. For the pump, its spectrum before the fiber input is shown in Fig. 4(a), and after the HNL-DSF output, the generation of supercontinuum is readily obvious as in Fig. 4(b). The dip around $1560 \mathrm{~nm}$ was due to the residual pump after the WDM coupler. Clearly observed from Fig. 4(b), the original spectrum of the SC was greatly broadened with the weak $\mathrm{CW}$ trigger, especially at some wavelength regions: $1380 \mathrm{~nm}$ to $1470 \mathrm{~nm}, 1630 \mathrm{~nm}$ to $1700 \mathrm{~nm}$ and beyond, which had significant enhancement. The largest power enhancement, occurred around $1400 \mathrm{~nm}$ and $1700 \mathrm{~nm}$, was as high as $40-\mathrm{dB}$ comparing to the non-trigger case. In order to investigate the quality of the wavelength conversion, different WDM couplers were used to slice out the SC spectrum from S-band $(1510 \mathrm{~nm})$ to L-band $(1615 \mathrm{~nm})$, which covered over $100 \mathrm{~nm}$. The insets of Fig. 4(b) show the wavelength converted signal at $1516.4 \mathrm{~nm}$ originated from pulsed pump, and the bandwidth of the VBTBPF was $3.2 \mathrm{~nm}$, and the time-domain pulse width of the wavelength converted signal was 13 ps (Fig. 4(b)). The improvement with trigger is obvious as shown in the eye-diagrams.

In addition to $1516.4 \mathrm{~nm}$, several more wavelengths (C-band: 1536.15 and $1543.68 \mathrm{~nm}$; L-band: 1566.82, 1592.8 and $1611 \mathrm{~nm}$ ) were measured across nearly 100-nm spectrum while keeping the same filter bandwidth. With the exception of S-band, all the recorded eye-diagrams and their corresponding BER at $10^{-9}$ are shown in Figs. 5 and 6. Stability improvement can be observed from the eye-diagrams for all measured wavelengths. Error-free operations were achieved with less than or equal to 3.8-dB power penalty. With proper filtering and detection equipment, it will also be interesting to further explore the red-shifted (around $1700 \mathrm{~nm}$ ) and blue-shifted 


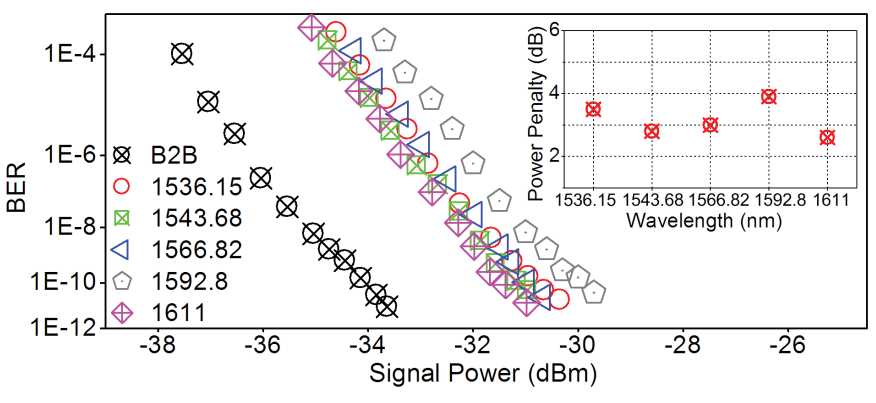

Fig. 6. BER curves and their corresponding power penalties.

(around $1400 \mathrm{~nm}$ ) region of the triggered SC spectrum. This part together with different modulation formats will be the focus of our future work.

\section{CONCLUSION}

We have demonstrated the pulse-to-pulse power stability improvement as well as the bandwidth enhancement of the CW-triggered SC scheme and utilized it for realizing wideband and error-free wavelength conversion. Specifically, we showed, to the best of our knowledge, for the first time that the rogue void condition of the SC can be transformed to the more stable (quasi-Gaussian) temporal statistics by introducing the $\mathrm{CW}$ trigger. In addition, the SC spectrum was significantly enhanced to nearly tripled bandwidth (wider than $300 \mathrm{~nm}$ ). In the wavelength conversion based on the $\mathrm{CW}$-triggering scheme clear and widely open eye-diagrams have been obtained for all wavelength points, from 1510 to $1615 \mathrm{~nm}$. While less than or equal to $3.8-\mathrm{dB}$ power penalty were achieved for C-band and L-band signals. The proposed scheme provides a low pump power and wide covering range solution for wavelength conversion. With better $\mathrm{CW}$ trigger source, the scheme could be further extended to wavelength conversion using the phase-modulated signals.

\section{ACKNOWLEDGMENT}

The authors would like to thank Sumitomo (SEI), Osaka, Japan, for providing the HNL-DSF.

\section{REFERENCES}

[1] R. R. Alfano, The Supercontinuum Laser Source: Fundamentals with Updated References, 2nd ed. New York: Springer-Verlag, 2005.

[2] J. M. Dudley, G. Genty, and S. Coen, "Supercontinuum generation in photonic crystal fiber," Rev. Mod. Phys., vol. 78, no. 4, pp. 1135-1184, 2006.

[3] J. Herrmann, et al., "Experimental evidence for supercontinuum generation by fission of higher-order solitons in photonic fibers," Phys. Rev. Lett., vol. 88, no. 17, pp. 173901-173904, 2002.

[4] D. R. Solli, C. Ropers, P. Koonath, and B. Jalali, "Optical rogue waves," Nature, vol. 450, pp. 1054-1057, Dec. 2007.

[5] D. R. Solli, C. Ropers, and B. Jalali, "Rare frustration of optical supercontinuum generation," Appl. Phys. Lett., vol. 96, no. 15, pp. 151108-151110, 2010.

[6] G. Genty, S. Coen, and J. M. Dudley, "Fiber supercontinuum sources (Invited)," J. Opt. Soc. Amer. B, vol. 24, no. 8, pp. 1771-1785, 2007.

[7] K. K. Y. Cheung, C. Zhang, Y. Zhou, K. K. Y. Wong, and K. K. Tsia, "Manipulating supercontinuum generation by minute continuous wave," Opt. Lett., vol. 36, no. 2, pp. 160-162, 2011.

[8] Q. Li, F. Li, K. K. Y. Wong, A. P. T. Lau, K. K. Tsia, and P. K. A. Wai, "Investigating the influence of a weak continuous-wave-trigger on picosecond supercontinuum generation," Opt. Express, vol. 19, no. 15, pp. 13757-13769, 2011.

[9] D. R. Solli, C. Ropers, and B. Jalali, "Active control of rogue waves for stimulated supercontinuum generation," Phys. Rev. Lett., vol. 101, no. 23, pp. 233902-233905, 2008.

[10] P. M. Moselund, M. H. Frosz, C. L. Thomsen, and O. Bang, "Backseeding of higher order gain processes in picosecond supercontinuum generation," Opt. Express, vol. 16, no. 16, pp. 11954-11968, 2008.

[11] A. S. Gouveia-Neto, M. E. Faldon, and J. R. Taylor, "Raman amplification of modulational instability and solitary-wave formation," Opt. Lett., vol. 13, no. 11, pp. 1029-1031, 1988.

[12] M. Scaffardi, F. Fresi, A. Bogoni, and L. Poti, "Implementation of a tunable $160 \mathrm{~Gb} / \mathrm{s}$ wavelength multi-converter based on supercontinuum in a highly nonlinear fibre," in Proc. ECOC 2006, pp. 1-2.

[13] O. F. Yilmaz, S. R. Nuccio, X. Wu, and A. E. Willner, "Tunable N-fold multicasting and pulsewidth of $40 \mathrm{~Gb} / \mathrm{s}$ channels by variable periodic slicing of a supercontinuum," in Proc. CLEO/QELS 2009, pp. 1-2.

[14] S. Oshiba and R. Moritomo, "All-optical arbitrary wavelength conversion with signal regeneration based on slicing of supercontinuum spectrum," in Proc. IEEE/LEOS Winter Topicals Meeting Ser, Jan. 2009, pp. $256-257$.

[15] X. Xu, C. Zhang, T. I. Yuk, K. K. Tsia, and K. K. Y. Wong, "Wide-band error-free wavelength conversion based on CW-triggered supercontinuum," in Proc. OFC/NFOEC 2012, Los Angeles, CA, pp. 1-3, paper OM3C.1.

[16] A. Kudlinski, B. Barviau, A. Leray, C. Spriet, L. Héliot, and A. Mussot, "Control of pulse-to-pulse fluctuations in visible supercontinuum," Opt. Express, vol. 18, no. 26, pp. 27445-27454, 2010.

[17] Y. Qiu, C. Zhang, K. K. Y. Wong, and K. K. Tsia, "Demonstration of minute continuous-wave triggered supercontinuum generation at $1 \mu \mathrm{m}$ for high-speed bio-photonic applications," Proc. SPIE Photon. West, vol. 8240, pp. 82400O-1-82400O-7, Dec. 2012. 